\title{
$K u$-Band Long Distance Site-Diversity (SD) Characteristics Using New Measuring System
}

\author{
Takeshi Hatsuda, Member, IEEE, Yoshinao Aoki, Member, IEEE, Hiroshi Echigo, Member, IEEE, \\ Fumio Takahata, Member, IEEE, Yasuyuki Maekawa, Member, IEEE, and Kiyotaka Fujisaki
}

\begin{abstract}
This paper deals with the short $(10 \mathrm{~km})$ and long distance $(300-1400 \mathrm{~km})$ site-diversity (SD) characteristics by using a newly developed measuring system. In the proposed measuring system, six earth stations transmit 14-GHz band QPSK signals, and one measuring earth station receives 12-GHz band signals and processes them to determine SD characteristics. As a result, easy operation and maintenance, low-cost measuring system construction and highly accurate data have been obtained. By comparing those measured results with the SD joint probability approximation equation in ITU-R Rec.P.618-7, a good agreement can be obtained. Furthermore, the effect of typhoons on SD characteristics were measured.
\end{abstract}

Index Terms - Rain attenuation, satellite communication system, site-diversity (SD) measurement system, SD characteristics, VSAT system design.

\section{INTRODUCTION}

$\mathbf{S}$ ATELLITE communication technologies are now highly developed, and high quality, distance-independent services have expanded over a very wide area. In particular, low cost VSAT networks for data, voice, and video communications have expanded widely with new services and new applications. The Hokkaido Integrated Telecommunications (HIT) network, as shown in Fig. 1, has been studied with satellite communication systems and low-cost super high frequency (SHF) band terrestrial radio networks. By using the HIT network, economical and highly functional services can be realized [1].

Other studies concerned with the HIT-Network were carried out in various areas, e.g., resolution of snow accumulation on antennas in snowy climates by using a snow melting antenna system [2] and a negative-gradient slanted polymer-shielded antenna [3]. Some interference problems from terrestrial radio relay networks are discussed by introducing the theoretical Desired signal to Undesired signal power ratio (D/U) value calculation formulas between fixed-satellite service and terrestrial radio relay service, and interference experiments have been conducted to obtain interference from 4 and $11 \mathrm{GHz}$ bands

Manuscript received January 8, 2002; revised July 16, 2003. This work was supported in part by Japan Satellite Systems, Inc., and in part by the Ministry of Education, Science and Culture of Japan under Grants-in-Aid for Scientific Research 06650427 and 09650420.

T. Hatsuda is with the Hokkaido Institute of Technology, Sapporo 006-8585 Japan (e-mail: hatsuda@ieee.org).

Y. Aoki is with Hokkaido University, Sapporo 060, Japan

H. Echigo is with Tohoku Gakuin University, Tagajo 985-8537, Japan.

F. Takahata is with Waseda University, Tokyo 165, Japan.

Y. Maekawa is with Osaka Electro-Communication University, Neyagawa 572, Japan.

K. Fujisaki is with Kyushu University, Fukuoka 812-8581, Japan.

Digital Object Identifier 10.1109/TAP.2004.829845

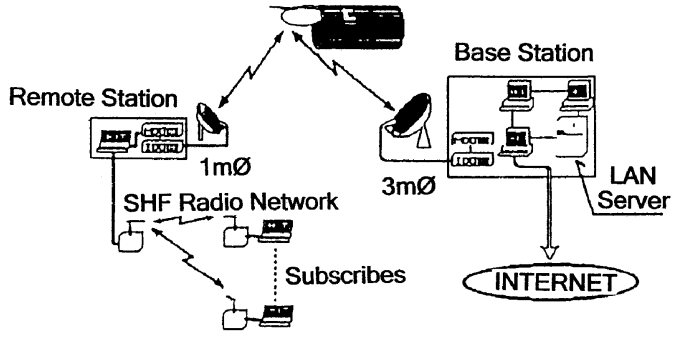

Fig. 1. HIT network concept.

terrestrial radio relay systems to fixed-satellite services by the using newly developed measuring system [4], [5].

As for the system design of the HIT network, it must first overcome outages of satellite links due to rain attenuation, which is the most difficult problem for the $14 / 12$ and $30 / 20$ $\mathrm{GHz}$ bands.

There are many researches with the aim of studying propagation characteristics in the earth-space link at 11, 12, 20, 30, and $50 \mathrm{GHz}$ bands [6]-[14]. Especially, in these works, there are great advantages about propagation characteristics by using high frequencies communication satellites, e.g., the Advanced Communication Technology Satellite (ACTS) conceived at the National Aeronautics and Space Agency (NASA) in the U.S., experimental communication satellite OLYMPUS by Europe Space Agency, ITALSAT program by the Italian Space Agency in Europe and Japanese Communication Satellite (CS-3) developed by Nippon Telegraph and Telephone Public Corporation (NTT) in Japan.

In [6] and [7], the ACTS Satellite carried 20.2 and $27.5 \mathrm{GHz}$ beacons and was used for measuring rain attenuations and rain rate distributions at many sites in the U.S.

In [8]-[10], by using OLYMPUS and ITALSAT, propagation researches in Europe have been carried out to verify the viability of $20-50 \mathrm{GHz}$ frequency bands for high capacity radio transmission.

In [11], $20 \mathrm{GHz}$ propagation experiments by using CS-3 had been carried out in many sites in Japan.

From those propagation experiments data, it can become clear that the $K u$ - and $K a$ bands suffer from heavy rain attenuation and adequate techniques to counteract rain attenuation should be considered.

In a conventional network, a large rain margin for atmospheric effects is included in the link budget, and the earth station (ES) antenna becomes large, making the system expensive. However, in a VSAT system, low-cost ES construction is one of the most important matters. 


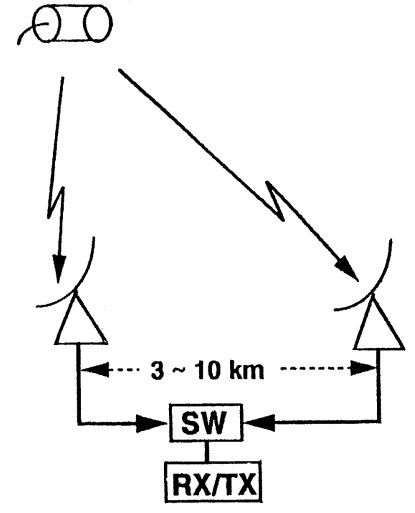

Fig. 2. SD concept.

There are various techniques to counteract rain attenuation as follows:

1) large rain margin;

2) bit rate reduction;

3) QPSK phase reduction;

4) site-diversity (SD);

5) satellite diversity (Sat. D).

The SD concept, as shown in Fig. 2, is one of the most promising fade countermeasure techniques. There are some SD data for specific distances and frequencies [12]-[22]. In [12], by using ETS-II satellite signals, rain attenuation characteristics for frequencies 11.5 and $34.5 \mathrm{GHz}$ bands and the correlation coefficient for distances within $10 \mathrm{~km}$ from 1977-1978 are analyzed. In [13], the simultaneous occurrence probabilities of rainfall obtained from 36 pair combinations among nine locations at distances from $400-600 \mathrm{~km}$ in Japan by using $12 \mathrm{GHz}$ broadcasting satellite (BS) signals are shown. [14] illustrates joint statistics of rain intensity in eight Italian cities of eight locations at distances from $150 \mathrm{~km}$. In [15], 50 locations and 10 years joint probabilities of rain attenuation outages occurring at two or more of the assisted stations are studied.

In [16]-[18], a three-site rain-fade space-diversity measurement has been carried out and examined the efficiency of employing three-site and two-site space-diversity systems to reduce the required fade margin. In [19]-[22], narrow angle diversity characteristics and rainy tropical region SD characteristics were studied.

However, SD will have different characteristics for different seasons, years, frequencies and topographical features, etc. Furthermore, in measuring SD characteristics, the synchronization methods, accuracies, simplicities, and low-cost measuring method must be considered.

In the future mobile satellite communication system, the use of satellite diversity (Sat. D) technique is envisaged [23]-[26]. When using this technique to improve the link availability, two co-located ESs establish a link with two satellites located in different orbital positions and the signal affected by the lower rain attenuation is selected. In such cases, long distance SD characteristics will be required for the design of Sat. D transponder sharing ratio [27]-[29].

The authors are members of the Digital Satellite Communication Universities Corporation Research Organization (Un-SAT), and multiple experiments have been carried out by using a satellite (JCSAT-1) transponder belonging to the Japan Satellite Systems Inc. New methods for measuring SD characteristics have been developed, in which six ESs transmit QPSK signals from six different areas and one measuring ES receives signals, which are processed to determine the SD characteristics [27]-[29]. In [27]-[29], a new method for the assessment of SD, based on six transmission ESs and one processing ES (that receives all QPSK signals transmitted by the other six ESs) is proposed. In [30], theoretical analyzes about $K u$ band rain and snow attenuation were carried out.

On the other hand, in countries where typhoons frequently pass (e.g., Japan), the effects of typhoons on SD characteristics must also be considered in relation to the link budget or availability design. Since typhoons remain for a few days over wide areas of Japan (100-500 km), the effect on the link budget cannot be neglected. Some measuring data are shown in [12], but the distance is within $10 \mathrm{~km}$.

In the following, short-distance $(10 \mathrm{~km})$, and long-distance (300-1400 km) SD characteristics are presented and compared with ITU-R Rec.P.618-7 theoretical values. The effects of typhoons on SD characteristics, compared with the Japan Weather Satellite (JWS) weather chart, were also measured [28].

\section{New Measurement Method}

In conventional methods for measuring SD characteristics, data measured at several receiving stations are transmitted by commercial exclusive transmission lines or stored in floppy disks and transmitted and processed by complex synchronization procedures in a processing center [13]. However, such methods have some problems with accuracy, synchronization errors, high costs of transmission and human resources, etc. as follows.

1) At each measuring point, an expensive spectrum analyzer and personal computer, associated accessories and data processing equipment, etc. will be necessary. The more measuring points, the higher the measuring equipment cost.

2) To process data from many measuring points, it is difficult to synchronize all parallel data. Real-time transmission can be realized by using exclusive commercial transmission lines, but the operation cost will increase significantly.

3) Human cost for measuring and maintenance of equipment must also be accounted for.

4) If a spectrum analyzer or the AGC output-voltage of an FM demodulator is used, errors due to the spectrum analyzer fluctuation, energy dispersal fluctuation, voltage drift etc. can occur. In this case, accurate data can not be obtained.

5) If a method for utilizing beacon signals is not available, a signal receiver or spectrum analyzer will be necessary. Some satellites (e.g., JCSAT-1) do not have beacon generators. In this case, another solution must be considered.

To resolve these problems, a new measurement method has been proposed [27]-[29]. In this new method, homogeneous 


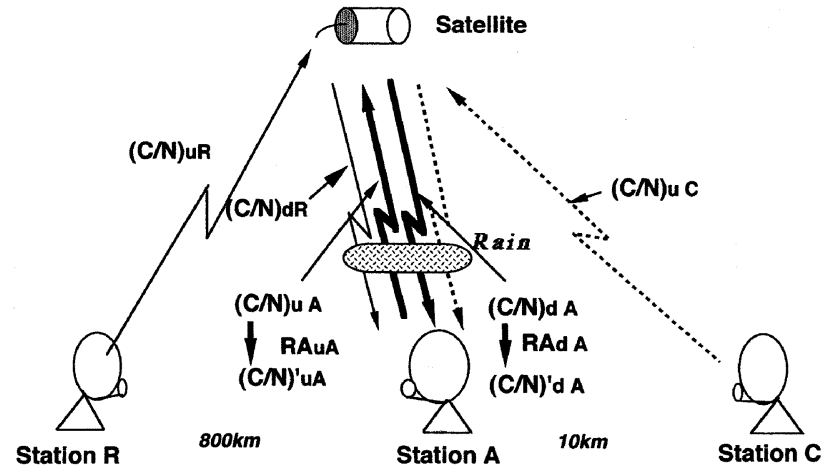

Fig. 3. Calculation model of rain attenuation and SD characteristics.

QPSK signals are received from several ESs and they are processed in one receiving ES.

In Fig. 3, earth station-A (ES-A) is the data processing earth station, and reference earth station (ES-R) and SD earth station (ES-C) are transmitting PSK signals. In Fig. 3, all experimental ESs have similar characteristics. Notations $u$ and $d$ mean up-link and down-link, and $A, C$ and $R$ indicate ES identification. The distance between $A$ and $R$ is $800 \mathrm{~km}$, which is sufficiently large to obtain good site diversity, and ES-R is assumed. The distance between stations $A$ and $C$ is approximately $10 \mathrm{~km}$.

\section{A. Determination of Rain Attenuation Using the ES-R Signal}

The experiments were carried out by the JCSAT-1 transponder. This satellite was not equipped with a beacon transmitter. Therefore, the ES-R signal was used for measuring attenuation signals.

1) Calculation Assumptions and Measured ESs Spectrum: To obtain more information about rain attenuation characteristics with wide measuring areas and long distance SD characteristics, a new calculation method is proposed and measurements were carried out. In the proposed method, two assumptions were used:

1) The ES-R does not encounter rain attenuation at the same time as measuring ES-A, because of the sufficiently large distance between the two ESs, and

2) All QPSK carriers have sufficient transponder output power back-off (i.e., about $22 \mathrm{~dB}$ ), so the up-link signals are linearly amplified by the satellite transponder and proportionally received by the receiving ES.

One example of three ESs (ES-A, ES-R, and ES-C) spectrum is shown in Fig. 4. As shown in this Fig. 4, all ES signal levels are perfectly equal levels. When rain attenuation occurred, the signal level linearly decreases on the spectrum analyzer display. Up-link and down-link linearity characteristics were confirmed by changing the transmission power and checking the linear decrement or increment of the received signal levels.

Concerning about the separation of up- and down-link attenuations, as shown in Fig. 4, it can be separated by using different frequency channels.

When rain attenuation occurs on ES-R (in Tokyo), the spectrum analyzer signal level from ES-R will decrease. On the contrary, the signal level from the measuring ES-A will not de-

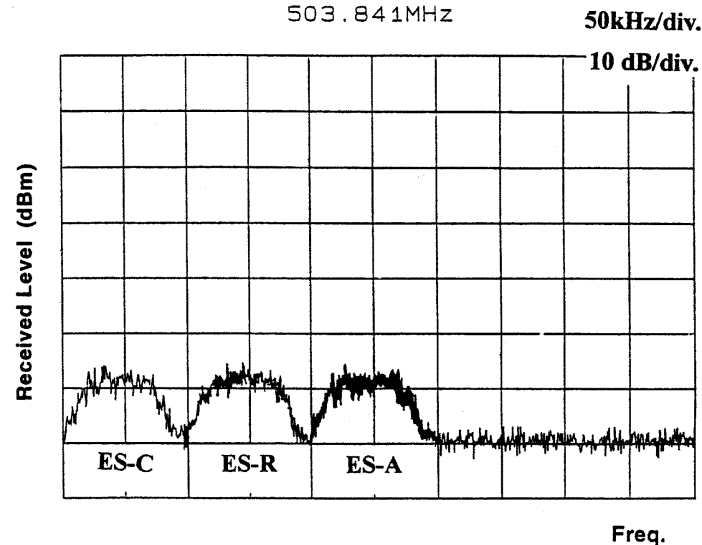

Fig. 4. Receiving signals example on spectrum analyzer from three ESs.

crease. By using this information, ES-R and ES-A signal attenuations can be separated.

2) Up- and Down-Link Rain Attenuation of ES-A: When rain attenuation occurred near the ES-A as shown in Fig. 3, this down-link attenuation is equal to the ES-R down-link attenuation. If the signal attenuation of ES-A and ES-R are defined as $R A_{d A}, R A_{d R}$, its values can be calculated by (1) and (2)

$$
\begin{aligned}
& R A_{d A}=\left(\frac{C}{N}\right)_{d A}-\left(\frac{C}{N}\right)_{d A}^{\prime}[\mathrm{dB}] \\
& R A_{d R}=\left(\frac{C}{N}\right)_{d R}-\left(\frac{C}{N}\right)_{d R}^{\prime}[\mathrm{dB}]
\end{aligned}
$$

where $(C / N)_{d A}$ and $(C / N)_{d R}$ are clear sky down-link $C / N$ values of the signal from ES-A and ES-R, and $(C / N)_{d A}^{\prime}$ and $(C / N)_{d R}^{\prime}$ are attenuated down-link $C / N$ values of ES-A and ES-R.

The down-link signal attenuation at station ES-A is the same for both signals, so

$$
R A_{d A}=R A_{d R}[\mathrm{~dB}]
$$

This means that the down-link attenuation of the ES-R signal level, which is measured by a spectrum analyzer in the ES-A, is equal to down-link attenuation of the ES-A.

Assuming that ES-R does not encounter rain attenuation at the same time as ES-A [assumption 1)], during attenuation on the signal from ES-A, the down-link attenuation $R A_{d R}$ is equal to the two-way (up- and down-links) attenuation of ES-R, $R A_{(u+d) R}$, which is measured by the spectrum analyzer.

The two-way (up- and down-links) attenuation of the ES-A is measured by the total attenuation of the ES-A signal level. Therefore

$$
\begin{aligned}
R A_{(u+d) A} & \\
= & \left\{\left(\frac{C}{N}\right)_{u A}-\left(\frac{C}{N}\right)_{u A}^{\prime}\right\}+\left\{\left(\frac{C}{N}\right)_{d A}-\left(\frac{C}{N}\right)_{d A}^{\prime}\right\} \\
= & \left(R A_{u A}+R A_{d A}\right)[\mathrm{dB}]
\end{aligned}
$$


where $(C / N)_{u A}$ is clear sky up-link $C / N$ value of the signal from ES-A, $(C / N)_{u A}^{\prime}$ is attenuated up-link $C / N$ values for ES-A.

From (1)-(4), the up-link attenuation of ES-A $R A_{u A}$ can be found as follows:

$$
R A_{u A}=R A_{(u+d) A}-R A_{d A}=R A_{(u+d) A}-R A_{d R}[\mathrm{~dB}] .
$$

This means that the up-link attenuation of ES-A can be derived as the signal level attenuation difference between ES-R and ES-A.

Similarly, the up-link attenuation of the ES-C $R A_{u C}$ can be determined by (6) as follows:

$$
R A_{u C}=R A_{(u+d) C}-R A_{d C}=R A_{(u+d) C}-R A_{d R}[\mathrm{~dB}] .
$$

This means that the up-link attenuation of ES-C can be established by the signal level attenuation difference between ES-C and ES-R.

From the results of up-link attenuations of $R A_{u A}$ and $R A_{u C}$, the SD characteristics between ES-A and ES-C can be obtained. In the method, only one ES with the lowest attenuation can be evaluated for site diversity characteristics.

\section{B. Determination of Rain Attenuation Using the Satellite Beacon Signal}

If the satellite is equipped with $a$ beacon transmitter in the same frequency band, those signals can be used instead of the reference signal of the ES-R, because its attenuation will be equal to the down-link attenuation $R A_{d A}$. The up-link attenuation can be determined by

$$
R A_{u A}=R A_{(u+d) A}-R A_{\text {beacon }}[\mathrm{dB}]
$$

where $R A_{\text {beacon }}$ is the attenuation value of the beacon signal in the ES-A, which is equal to the down-link attenuation $R A_{d A}$.

\section{Determination of Rain Attenuation Using Up-and Down-Link Signal Attenuation of ES-A}

On the long distance link from ES-R to ES-A, rain attenuation will occur on the up-link and down-link simultaneously with a small probability in a special case, e.g., the passage of a typhoon. In such cases, the results of the assumption made in Section II-A will not be correct. In this case, down-link attenuation of the ES-R signal can not utilized.

One solution is the utilization of the relation between up- and down-link attenuations[30], [31].

1) Measured Result Examples: Fig. 5 shows an example of the relation between down-link attenuation from ES-R $R A_{d R}$ and both-way attenuation of ES-A $R A_{(u+d) A}$. The measurement was carried out by using a $14.003 \mathrm{GHz}$ transmitting frequency and $12.255 \mathrm{GHz}$ receiving frequency of a JCSAT transponder.

The relation between $R A_{d R}$ and $R A_{(u+d) A}$ is obtained by the least square method as follows:

$$
R A_{d R}=0.498 \times R A_{(u+d) A}+0.087[\mathrm{~dB}] .
$$

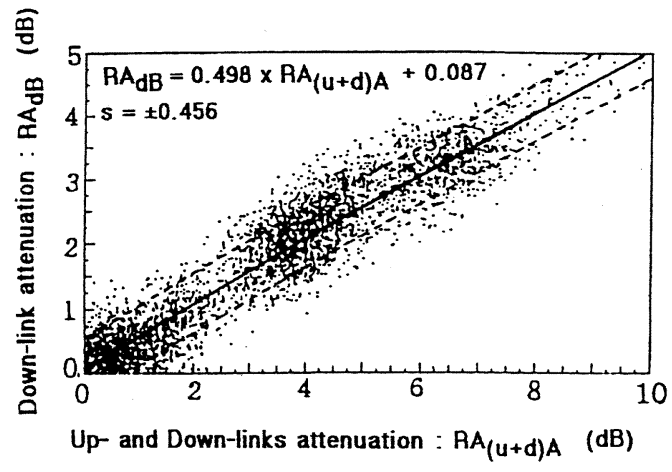

Fig. 5. Relations between both links rain attenuation and down-link rain attenuation characteristics.

The dotted lines in Fig. 5 show standard deviation values, and $\underline{\sigma}= \pm 0.46[\mathrm{~dB}]$. This value is less than twice the spectrum analyzer measuring error $( \pm 0.25 \mathrm{~dB})$. Equation (8) means that half the value of $R A_{(u+d) A}$ is approximately equal to the up-link attenuation and the down-link attenuation.

2) Theoretical System Noise Temperature Considerations: According to ITU-R Rec.P.838-1 [33], the relationship between specific rain attenuation $\gamma_{R}[\mathrm{~dB} / \mathrm{km}]$ and rain rate $R[\mathrm{~mm} / \mathrm{h}]$

$$
\gamma_{R}=k R^{\alpha}[\mathrm{dB} / \mathrm{km}]
$$

where $k, \alpha$ : parameters depend on frequency and polarization, respectively.

For linear and circular polarization, and for all path geometries, the coefficient in (9) can be calculated from the values in Table I in ITU-R Rec.P.838-1 using

$$
\begin{aligned}
k & =\frac{\left[k_{H}+k_{V}+\left(k_{H}-k_{V}\right) \cos ^{2} \theta \cos 2 \tau\right]}{2} \\
\alpha & =\frac{\left[k_{H} \alpha_{H}+k_{V} \alpha_{V}+\left(k_{H} \alpha_{H}-k_{V} \alpha_{V}\right) \cos ^{2} \theta \cos 2 \tau\right]}{2 k}
\end{aligned}
$$

where $\theta$ is the path elevation angle and $\tau$ is the polarization tilt angle relative to the horizontal.

The rain attenuation: $A$ is

$$
A=\int_{0}^{D} \gamma_{R} d x[\mathrm{~dB}]
$$

where $D$ is the slant path length.

Furthermore, the increase due to noise of the low noise amplifier must be considered [30], [32].

This is because at ES-A, the attenuation is estimated as the difference of the received $C / N$ with respect to the clear-sky $C / N$ [see (1)]. The measured attenuation is thus increased by the increase of the sky noise in the down-link frequency band during rain.

The system noise temperature: $T_{\text {sys }}$ can be described by the following equation:

$$
\begin{aligned}
T_{\mathrm{sys}} & =\frac{\left(T_{a}+T_{s}\right)}{L}+T_{p}\left(\frac{1-1}{L}\right)+T_{r} \\
T_{s} & =T_{m}-\frac{T_{m}}{10^{A / 10}}
\end{aligned}
$$




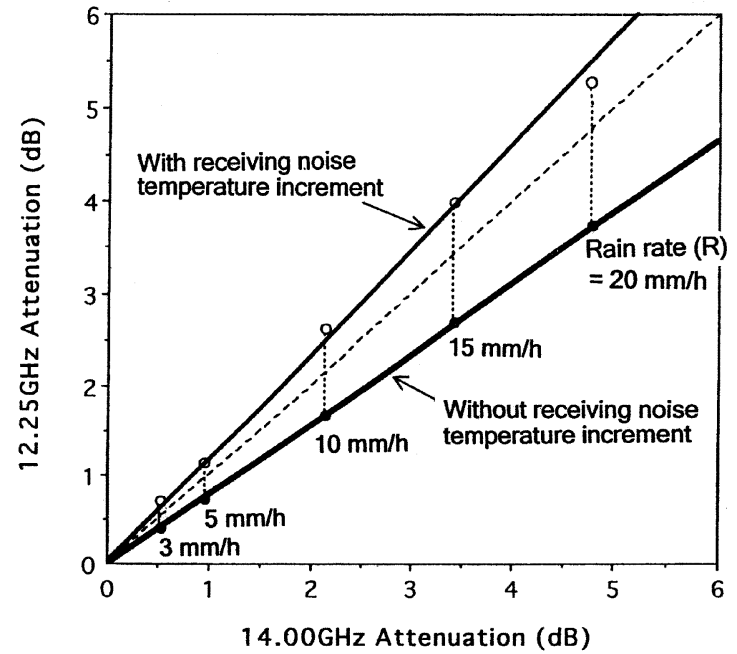

Fig. 6. Relations between $14.00 \mathrm{GHz}$ band link rain attenuation and 12.25 $\mathrm{GHz}$ band link rain attenuation characteristics.

where $T_{a}$ : the antenna noise temperature $[K], T_{s}$ : the sky noise temperature $[K], T_{p}$ : the ambient temperature of antenna feeder $[K], L$ : the loss of feeder, $T_{r}$ : the LNA noise temperature $[K]$, $T_{m}$; the average absorption temperature of the atmosphere $\left(T_{m}=255.7 \mathrm{~K}\right)$.

Fig. 6 shows the calculated results of (9)-(14), with $T_{a}=$ $25 \mathrm{~K}, T_{p}=293 \mathrm{~K}, L=0.5 \mathrm{~dB}, T_{r}=120 \mathrm{~K}$. As shown in Fig. 6 , the attenuation at $14 \mathrm{GHz}$ is similar to the down-link attenuation at $12 \mathrm{GHz}$ plus the noise increment.

The measured data and theoretical results show that almost the same attenuation values are obtained for up- and down-links due to the $12 \mathrm{GHz}$ band receiver noise. Therefore, the up-link attenuation of ES-A: $R A_{u A}$ can be found as follows, combining (5) with (8):

$$
\begin{aligned}
R A_{u A} & =\frac{R A_{(u+d) A}-\left(R A_{(u+d) A}\right)}{2} \\
& =\frac{\left(R A_{(u+d) A}\right)}{2}[\mathrm{~dB}] .
\end{aligned}
$$

Similarly, the up-link attenuation of ES-C: $R A_{u C}$ can be found as follows,

$$
R A_{u C}=\frac{R A_{(u+d) C}-\left(R A_{(u+d) A}\right)}{2}[\mathrm{~dB}] .
$$

From the results of up-link attenuations $R A_{u A}$ and $R A_{u C}$, the SD characteristics between the ES-A and ES-C can be obtained without using the ES-R or the beacon signal.

\section{Measuring Accuracy Consideration Using the ES-R Signals}

The distance between measuring ES-A (Hokkaido Institute of Technology, Sapporo, Japan) and ES-R (Waseda University, Tokyo, Japan) is about $800 \mathrm{~km}$. At such long distances, the joint probability for rain attenuation events at ES-A and ES-R can be estimated about $10^{-2}-10^{-4}$ from ITU-R Rec. P.618-7 [34] and measured data of this work. This means that simultaneous rain attenuation occurrence would be a very small percentage of the year.

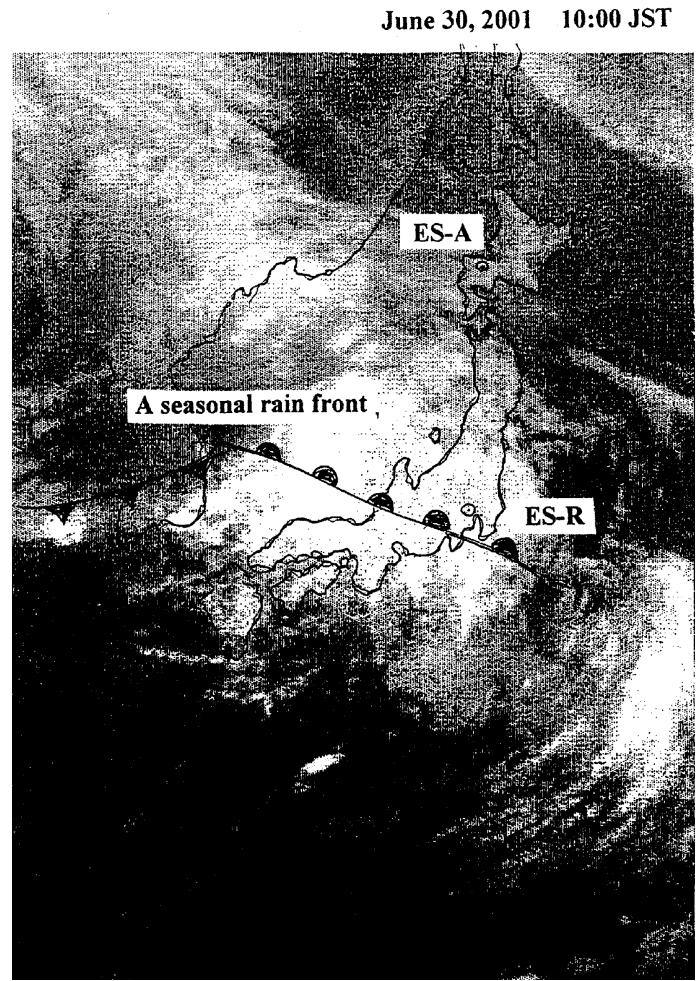

Fig. 7. Typical weather map photograph from weather satellite in the rainy season near Japan (ES-R suffers from attenuation, however, ES-A does not).

Furthermore, the following unique climate characteristics in Japan should be considered as reducing further the probability of simultaneous rain attenuation events between ES-A and ES-R:

1) In Japan, the rainy season ("Tsuyu" in Japanese) comes in June and July in the main islands (where Tokyo is located), but not in Hokkaido in the northern part of Japan (where ES-A in Sapporo is located). A typical weather photograph from a weather satellite in the rainy season is shown in Fig. 7. Hokkaido does not suffer from rain attenuation in this season. Therefore, in the rainy season of Japan in June and July, rain attenuation events measured at the link ES-R-ES-A are very likely to be present at ES-R rather than at ES-A.

2) On the contrary, in the winter season, ES-A in Sapporo suffers from signal attenuation due to very heavy snowfall. ES-R in Tokyo, however, has generally clear weather and does not suffer from heavy rain attenuation in this season, as shown in Fig. 8.

3) Even though typhoons pass through much of the main land of Japan, it is very rare that they cause rain attenuations at ES-A and ES-R simultaneously because the diameter of typhoon clouds in Japan ranges from 100 to 500 $\mathrm{km}$.

Due to those special climate characteristics and the long separation distance $(800 \mathrm{~km})$ between ES-A and ES-R, the measuring accuracy will be better than that of other measuring combinations, e.g., ES-A and station in the same region in the Honshu area, or short distance ES combinations. 


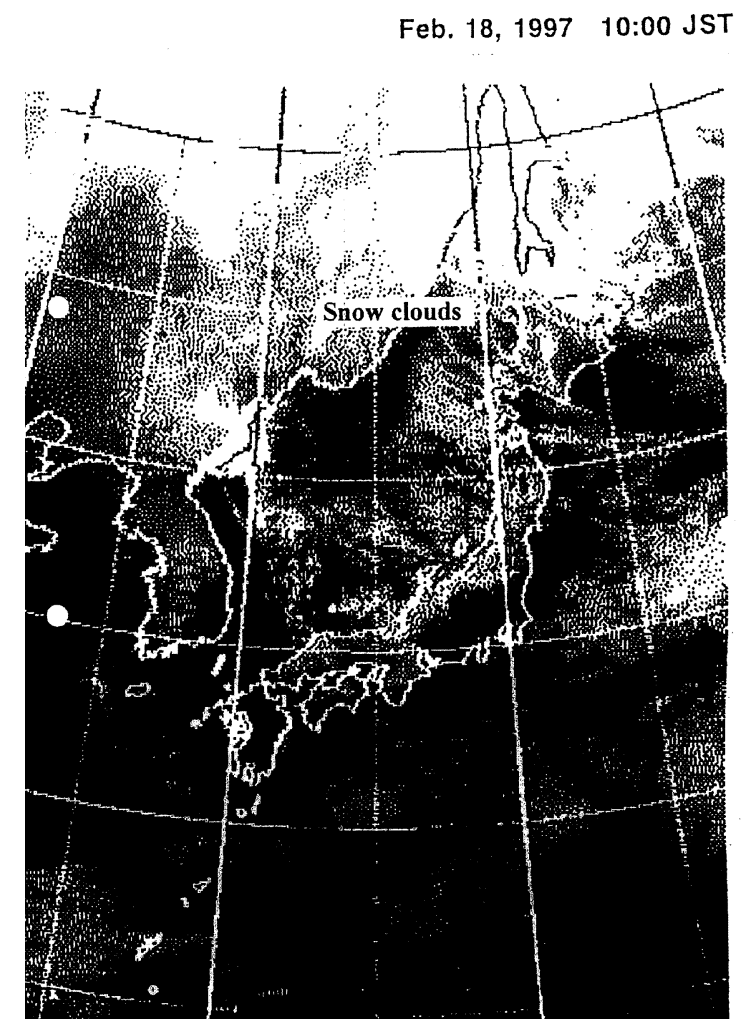

Fig. 8. Typical weather map photograph from weather satellite in winter season near Japan (ES-A suffers from snow-cloud attenuation, however, ES-R does not).

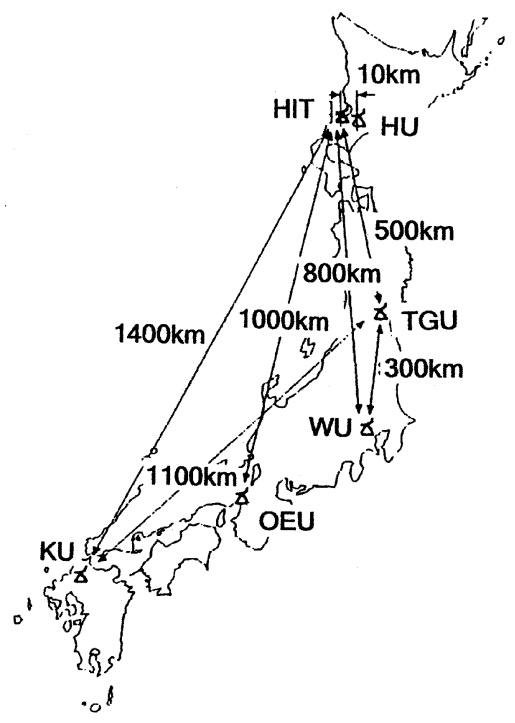

Fig. 9. Position of measuring ESs.

The measured JCSAT satellite was not equipped with beacon transmitter, however, if the beacon transmitters are equipped in the JCSAT satellite, measuring procedures become simpler and higher measuring accuracy could be accomplished.

\section{RESUltS OF MEASUREMENTS}

To obtain SD characteristics, six ESs, locations of which are shown in Fig. 9, transmit $64 \mathrm{~kb} / \mathrm{s}$ QPSK signals at $14 \mathrm{GHz}$ up-

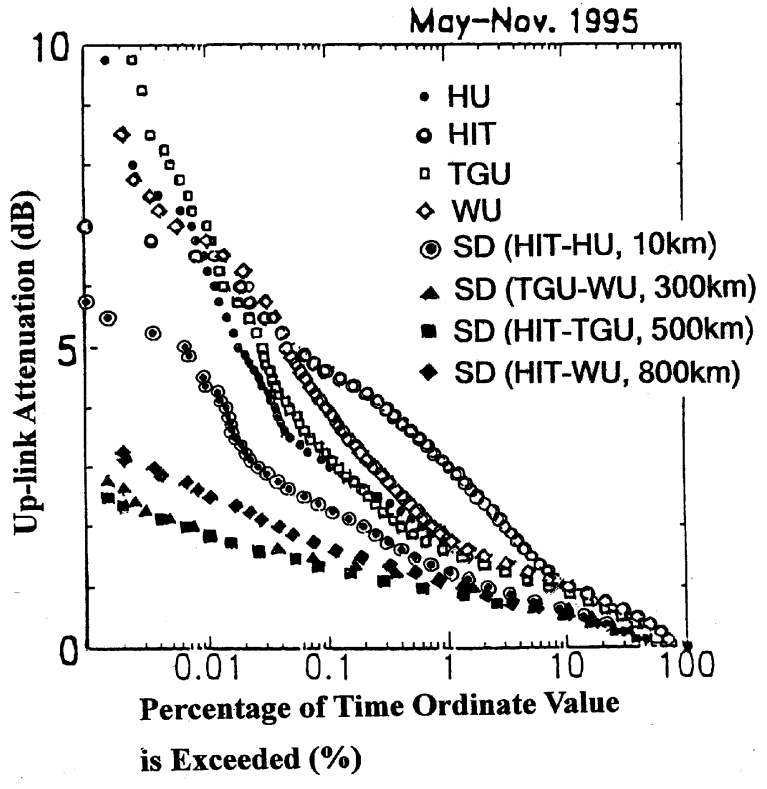

Fig. 10. Up-link rain attenuation and SD characteristics for experimental ESs.

wards, and ES-A receives the down-link signals at $12.25 \mathrm{GHz}$. In Fig. 9, HIT means the ES of the Hokkaido Inst. of Tech., HU means the Hokkaido Univ., TGU means the Tohoku Gakuinn Univ., WU means the Waseda Univ., OEU means the Osaka Electric Univ. and KU means the Kyusyuu Univ., respectively. The received signals are processed by personal computer (PC). The PC also controls the spectrum analyzer with sampling and averaging procedures. The measured data are stored on floppy disks or hard disks.

In order to reduce satellite transponder nonlinearity, the occurrence of signals lower than transponder back-off levels (20 $\mathrm{dB}$ ) is checked.

\section{A. Short (10 Km) and Long (300-1400 Km) Distance Up-Link SD Characteristics}

Fig. 10 shows for short $(10 \mathrm{~km})$ and long $(300-800 \mathrm{~km})$ distance up-link SD the relations between up-link attenuation and percentage of time that the ordinate value is exceeded. The measured interval is from May to November in 1995. The measured down-link frequencies are from 12.255 to $12.257 \mathrm{GHz}$, while the SD up-links are at $14.003 \mathrm{GHz}$. From analyzing Fig. 10, the following observations can be made.

1) By introducing the SD system, the outage probability is 10 times smaller than without the SD system for a short-distance $(10 \mathrm{~km})$, and a diversity gain (which defined the difference (in decibels) between the single-site attenuation and diversity attenuation values for the same time percentage) of $1.5 \mathrm{~dB}$ can be obtained for $0.01 \%$ of the time.

2) For a long-distance $(300-800 \mathrm{~km})$ the outage probability is 100-1000 times smaller than without the SD system, and a diversity gain of approximately $5 \mathrm{~dB}$ can be obtained for $0.01 \%$ of the time.

3) The improvement factors (which defined as the ratio of the lowest single-site time percentage and diversity time percentage at the same attenuation level) by the 


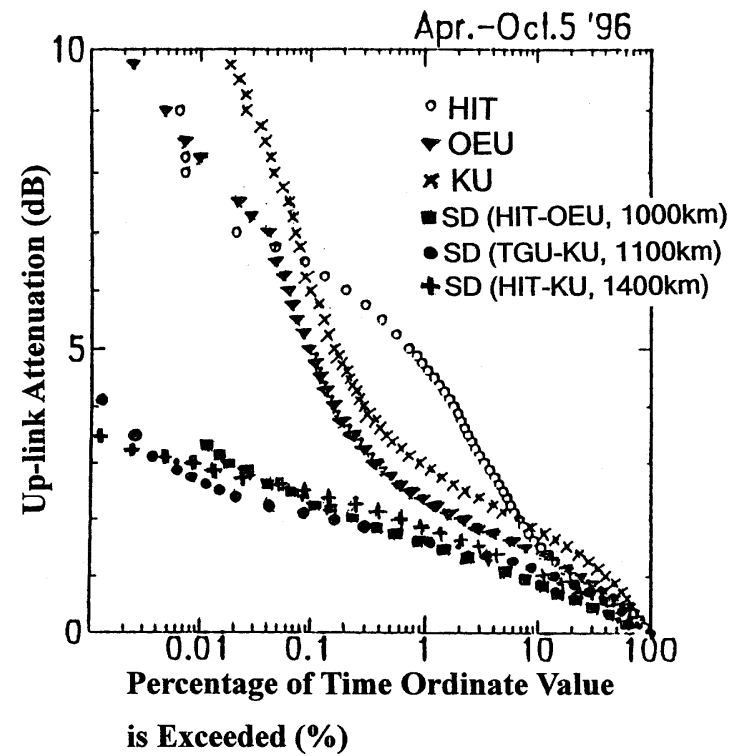

Fig. 11. Up-link rain attenuation and SD characteristics for $1000-1400 \mathrm{~km}$ experimental ESs.

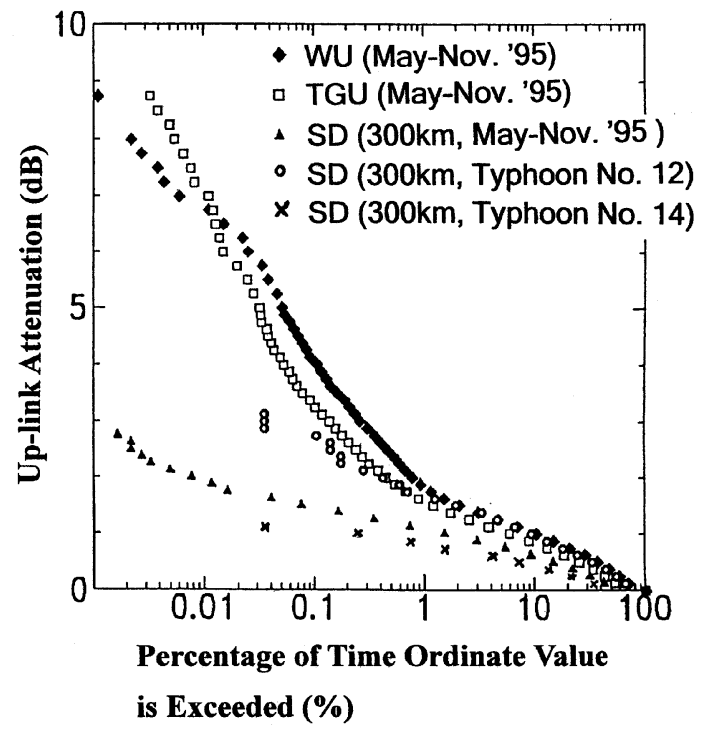

Fig. 12. Up-link rain attenuation and SD characteristics and effects from typhoon Nos. 12 and 14.

SD system are remarkable for ESs in climate areas with large rain attenuation.

Fig. 11 shows an example of 1000-1400 km distance SD characteristics between Sapporo and Osaka and between Sapporo and Fukuoka.

The measured interval is from April to October in 1996. The measurement periods of the statistics contained in Figs. 10 and 11 differ by almost one year because of ES construction delays and budget reasons.

As shown in Fig. 11, these SD characteristics are almost the same as the $300-800 \mathrm{~km} \mathrm{SD}$ characteristics.

\section{B. SD Results and Effects From Typhoons 12 and 14 in 1996}

In Fig. 12, an example of the long-term up-link SD characteristics for the ESs of Waseda Univ. and Tohoku Gakuin Univ. (distance of $300 \mathrm{~km}$ ) are shown as " $\mathbf{\Delta}$ " marks (also represented in Fig. 10 by the TGU-WU line). Other curves ( $O$ and $x$ ) show the effects from typhoons 12 and 14, both of which occurred in September in 1996.

Typhoon 12 passed from the Pacific Ocean to central Japan, as shown in Fig. 13. A cloud image of the typhoon transmitted from the weather satellite "Himawari" is shown in Fig. 14.

In this case, the ESs in Tokyo and Sendai (distance of $300 \mathrm{~km}$ ) are covered within the typhoon area. Due to this, the improvement is degraded as shown by " $O$ " marks in Fig. 12. The measurement time period covered approximately four days, starting from the day before the typhoon approach until the day after it had passed over the main land of Japan.

Typhoon 14 approached from the southern part of Japan, traversed the Japanese Sea, and passed over Sapporo, as shown in Fig. 13. In this case, Tokyo and Sendai did not suffer from the typhoon. As a result, the effects of the typhoon on site diversity statistics were relatively small, as shown by " $x$ " marks in Fig. 12.

From the measured results, the following points becomes clear.

1) By using the new measuring system, the influence of typhoons on long-distance SD characteristics was newly obtained. This method can be expanded to wider areas of Japan.

2) From SD characteristics is found that the outage probability using SD is 10 to 1000 times smaller than without SD.

3) Due to Typhoon 12, the outage probability reduction by SD was degraded 10 to 100 times with respect to the averaged (seven months) SD characteristics.

On the contrary, during Typhoon 14, the outage probability reduction by SD was about the same as the averaged (seven months) SD characteristics. This shows that the effect of typhoon on SD characteristics can be very variable.

In general, a typhoon covers a relatively wide area of Japan and remains over Japan for several days. Therefore, system designers must take availability degradation due to typhoons into account.

\section{THEORETICAL CONSIDERATIONS AND MEASUREMENT ACCURACY}

\section{A. SD Characteristics Comparison Between ITU-R Rec. P.618-7 and Measured Results}

The SD characteristics have been studied in ITU-R (former CCIR), e.g., Rec. P.618-7 [35]. In Rec. P.618-7, the diversity improvement factor, $I$, by introducing the SD system is expressed as follows:

$$
I=\frac{p^{1}}{p^{2}}=\left[\frac{1}{\left(1+\beta^{2}\right)}\right] \cdot\left[\frac{1+\beta^{2}}{p_{1}}\right] \approx 1+\left(\frac{100 \beta^{2}}{p_{1}}\right)
$$

where $p_{1}$ and $p_{2}$ are respective single-site and diversity time percentages, and $\beta$ is a parameter depending on link characteristics. 


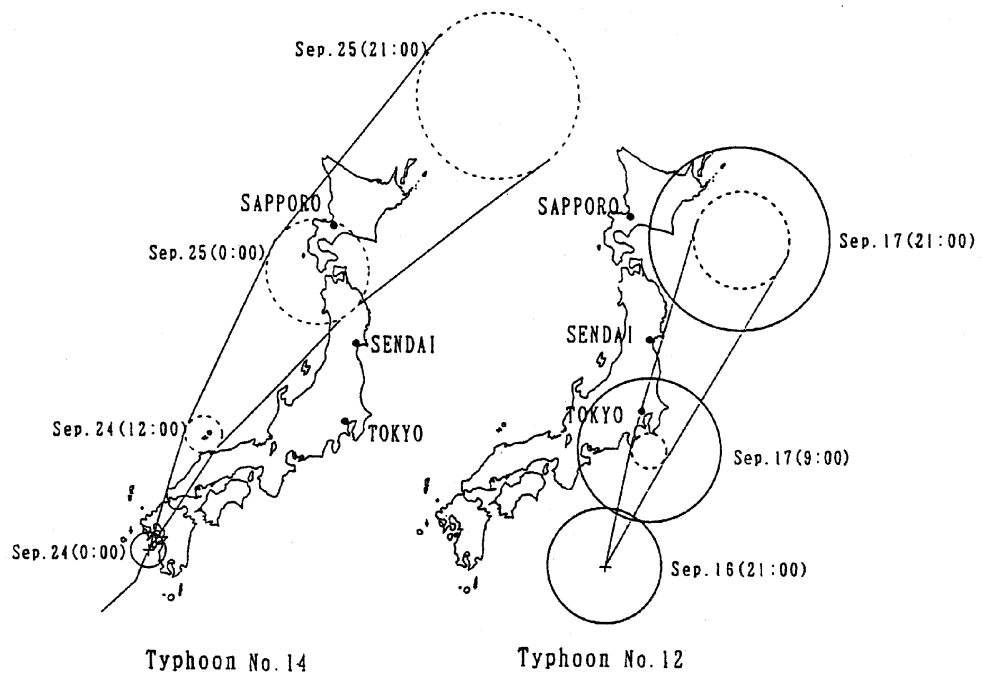

Fig. 13. Typhoon Nos. 12 and 14 passing roots.

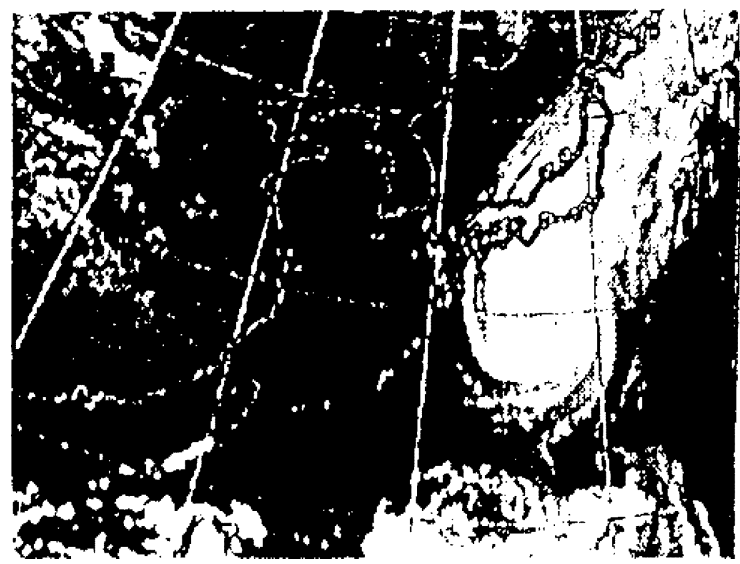

Fig. 14. Photograph of typhoon Nos. 12 from GMS satellite.

In Rec. P.618-7, it is described that the value of $\beta^{2}$ depends basically on the distance $d$ between the stations, and only slightly on the angle of elevation and the frequency.

The value of $\beta^{2}$ can be expressed by (18)

$$
\beta^{2}=10^{-4} \times d^{1.33}
$$

where $d$ is the separation distance (kilometers).

It is described in old CCIR Rep. 564-4 that (17) and (18) are in good agreement within $100 \mathrm{~km}$ with measured data in many countries. In the ITU-R Rec. P.618-7, the relationship between percentages of time with and without diversity for the same attenuation (Earth-satellite paths) is given for distance from $0 \mathrm{~km}$ to $50 \mathrm{~km}$.

To confirm the validity of (17) and (18) for more than $100 \mathrm{~km}$ SD distance, a comparison between experimental data (distances of 10-1400 km) and theoretical SD characteristics from (17) and (18) was carried out.

The diversity time percentages are plotted in Fig. 15, and the following become clear;

1) The experimental data and values obtained from (17) and (18) are in a relatively good agreement. This means that (17) and (18) can be expanded to approximately $1400 \mathrm{~km}$;

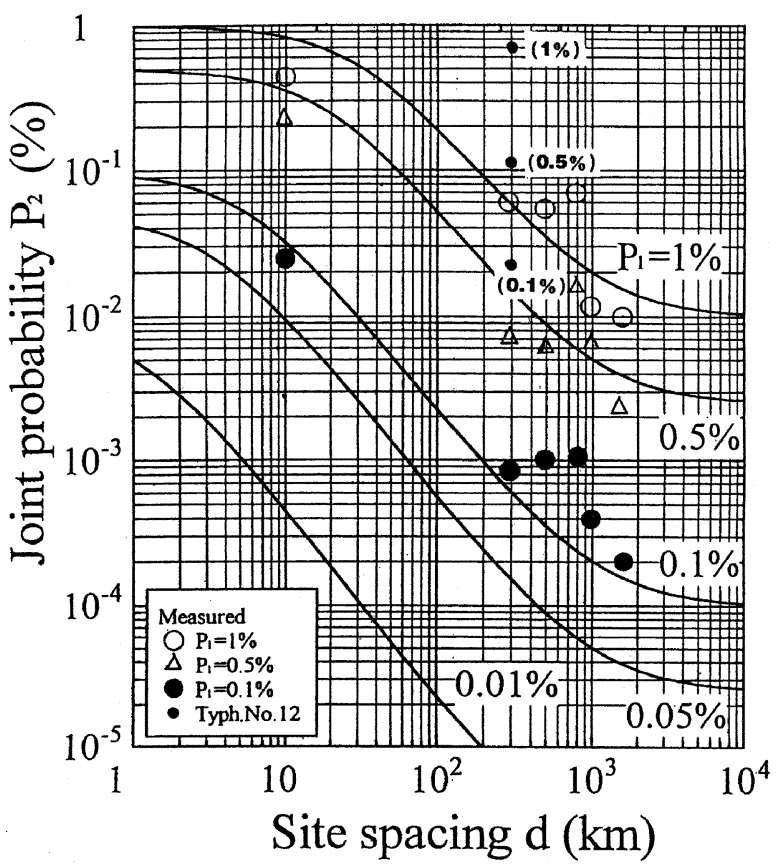

Fig. 15. SD effects for probability of up-link attenuation being simultaneously at two diversity ESs as a function of distances.

2) If wide areas are covered by clouds, e.g., passage of a typhoon, the diversity improvement factors will degrade. This factor must be considered in the design of the SD system.

\section{B. Assessment of Measurement Accuracy}

To assess the measurement accuracy, the following factors should be considered.

1) The main uncertainty in the measurement accuracy is probability of simultaneous attenuation at ES-A and ES-R. As described above, this probability is small, due to the long distance between ES-A and ES-R, and the special climate characteristics between ES-A and ES-R. This uncertainty of measurement accuracy can be 


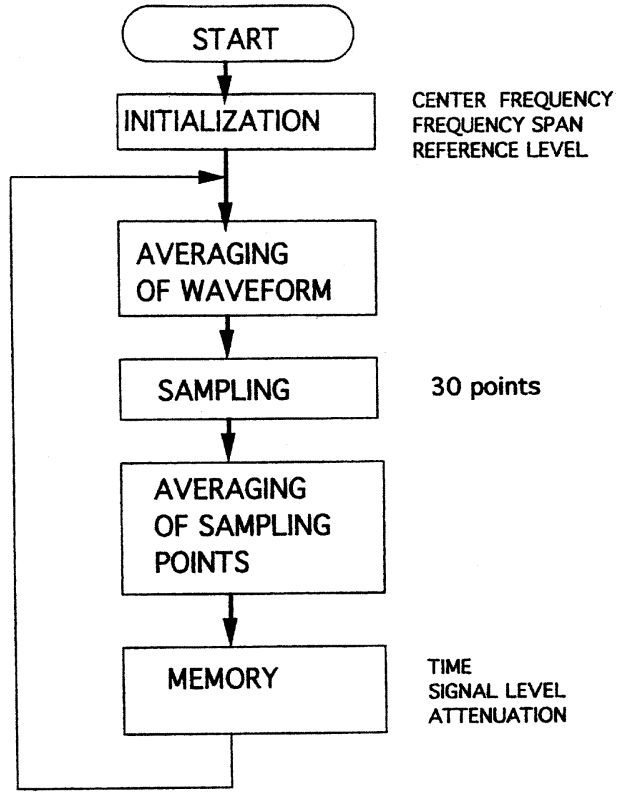

Fig. 16. Flow chart for measured data processing.

reduced further, using the two ES-R method, because joint probability of attenuation at three ESs is smaller than at two ESs simultaneously.

2) The measuring equipment (i.e., spectrum analyzer) accuracy will be approximately $\pm 0.25 \mathrm{~dB}$ according to the equipment specification.

3) The received signals, processed by the spectrum analyzer, are affected by noise, which causes amplitude fluctuations. To reduce those fluctuations, the signals were filtered using the averaging and sampling functions of the spectrum analyzer as shown in Fig. 16. Using this method, fluctuations can be reduced to up to $\pm 0.25 \mathrm{~dB}$.

4) The received signals are affected by fluctuations of the propagation parameters along the path (e.g., due to changes of drop size distribution) that cause amplitude fluctuations. As shown in Fig. 5, the resulting standard deviation values of those fluctuations are about $\pm 0.46 \mathrm{~dB}$.

5) Other factors affecting measurement accuracy like satellite transponder power change, ES transmitting power change, ES LNA characteristics fluctuation etc. can be taken into account. However, these effects are assumed to be small compared with the above mentioned other factors.

6) Accordingly, the accuracy can be roughly estimated to be within $\pm 0.5- \pm 1.0 \mathrm{~dB}$.

\section{CONCLUSION}

From a distance of $10-1400 \mathrm{~km}$, SD characteristics can be obtained by using the newly developed measuring method. In this measuring method, multiple ESs transmit QPSK signals and one receiving ES receives the down-link signals and processes them to determine SD characteristics.
Experiments were carried out using the JCSAT transponder and six ESs from northern to southern Japan, separated by distances of $10-1400 \mathrm{~km}$.

From the experimental results, the following SD characteristics can be clarified.

1) For a short-distance $(10 \mathrm{~km}) \mathrm{SD}$ system, the outage probability is about 10 times smaller than without the SD system.

2) For a long-distance (300-1400 km) SD system the outage probability is about 100 to 1000 times smaller than without the SD system.

3) The measured SD data were compared with values obtained from the SD joint probability approximation equation in the ITU-R Rec.P.618-7, and a relatively good agreement is found for up to $1400 \mathrm{~km} \mathrm{SD}$ characteristics. From this comparison, it becomes clear that the approximation equation can be extended to long-distance SD characteristics. However, this experiment was carried out only in Japan, so for more general confirmation of the ITU-R Rec.P.618-7, experiments should last for a longer period and should be performed in regions with different climates.

4) The influence of the passage of a typhoon on SD characteristics was investigated. If the coverage area of the typhoon is very large, the time probability improvement factors by SD were 10-100 times lower than the averaged (seven months) SD characteristics.

5) Experimental data were measured by using a system with one receiving ES and multiple transmitting ES. This way, synchronization problems between SD data measured by multiple ESs can be overcome.

6) The uncertainty whether measured attenuation occurs on the up-link or down-link of the link ES-R to ES-A is reduced by the relative long distance (approximately 800 $\mathrm{km}$ ) between ES-R and ES-A, and the seasonal rain probability differences between ES-R and ES-A. However, utilization of a beacon signal is preferable, because of smaller ambiguities compared with the method utilizing the ES-R.

By the newly proposed measuring method, easy operation and low-cost SD measurements can be achieved. Further SD data measurements, e.g., snowfall period characteristics and medium distance SD should be continued.

\section{REFERENCES}

[1] T. Hatsuda, "Packet communication ultra-small aperture terminal system for the Hokkaido integrated telecommunication network," IEEE Trans. Microwave Theory Tech., vol. 43, pp. 1692-1698, July 1995.

[2] T. Hatsuda, H. Aoki, T. Ogawa, and S. Isikawa, "Satellite receiving problems in snow and some resolutions-snow melting antenna systems," IEICE Trans., vol. J74-B-II, no. 12, pp. 714-717, 1991.

[3] T. Hatsuda, S. Mizuno, K. Masuzuka, H. Nisi, and T. Ogawa, "Satellite receiving problems in snow climates and some resolutions-the indoor reception method and the negative-gradient slatted polymer shielded antenna," IEICE Trans., vol. J74-B-II, no. 12, pp. 718-722, 1991.

[4] T. Hatsuda, "Theoretical and measuring results of interference between fixed-satellite and terrestrial radio network in urban and rural areas at 4 and $11 \mathrm{GHz}$ bands," in Proc. Asia Pacific Microwave Conf., vol. 12.5, 1992, pp. 255-258. 
[5] T. Hatsuda and Y. Motozumi, "Theoretical and measuring results of interferences between fixed-satellite and terrestrial radio relay services at 4 and $11 \mathrm{GHz}$ bands," IEEE Trans. Aerosp. Electron. Syst., vol. 34, no. 1, pp. 23-32, 1998.

[6] R. Bauer, "Ka-Band propagation measurement: An opportunity with advanced communication technology satellite (ACTS)," Proc. IEEE, vol. 85, pp. 853-862, June 1997.

[7] R. K. Crane, X. Wang, D. B. Westenhaver, and W. J. Vogel, "ACTS propagation experiment: Experiment design, calibration, and data propagation and archieval," Proc. IEEE, vol. 85, pp. 863-878, June 1997.

[8] B. R. Arbesser-Rastburg and G. Brussaard, "Propagation research in Europe using the OLYMPUS satellite," Proc. IEEE, vol. 81, pp. 865-875, June 1993.

[9] F. Dintelmann, G. Ortgies, F. Rücker, and R. Levis, "Results from 12- to 30-GHz German propagation experiments carried out with the OLYMPUS satellite," Proc. IEEE, vol. 81, pp. 876-884, June 1993.

[10] B. R. Arbesser-Rastburg and Paraboni, "European research on Ka-band path propagation," Proc. IEEE, vol. 85, pp. 843-852, June 1997.

[11] Y. Karasawa and Y. Maekawa, "Ka-Band earth-space propagation research in Japan," Proc. IEEE, vol. 85, pp. 821-842, June 1997.

[12] Y. Furuhama and T. Ihara, "Propagation characteristics of millimeter and centimeter waves of ETS-II classified by rainfall types," in URSI Comm. F Symp., Quebec, Canada, 05, 1980, pp. 26-30.

[13] Y. Otsu, Y. Takahashi, and T. Casey, "Simultaneous occurrence probabilities and rainfall among nine locations in Japan," Electron. Lett., vol. 22, no. 18 , pp. 937-938, 1986.

[14] F. Barbaliscia and A. Paraboni, "Joint statics of rain intensity in eight italian location for satellite communication networks," Electron. Lett., vol. 18 , pp. 118-119, 1982.

[15] F. Barbaliscia, G. Ravaioli, and A. Paraboni, "Characteristics of the spatial statistical dependence of rainfall rate over large areas," IEEE Trans. Antennas Propagat., vol. 40, pp. 8-12, Jan. 1992.

[16] J. Goldhirsh, B. H. Musiani, A. W. Dissanayake, and K.-T. Lin, "Three-Site space-diversity experiment at $20 \mathrm{GHz}$ using ACTS in the eastern united stated," Proc. IEEE, vol. 85, pp. 970-980, June 1997.

[17] H. Helmken, R. E. Henning, J. Feil, L. J. Ippolino Jr, and C. E. Vogel, "A three-site comparison of fade-duration measurments," Proc. IEEE, vol. 85, pp. 917-925, June 1997.

[18] J. Goldhirsh and B. H. Musiani, "Two years of three site diversity measurement at $20 \mathrm{GHz}$ with ACTS," in Proc. USNC/URSI Nat Radio Science Meeting, sec. 101, July 7, 1997, pp. 452-452.

[19] C. Emrich and R. J. Acosta, "Narrow angle diversity research using acts Ka-band signal with two USAT ground stations," in Proc. USNC/URSI Nat. Radio Science Meeting, sec. 25, July 7, 1999, pp. 40-40.

[20] A. Rochen and J. Neves, "Moderate rain rate characterization for small fade margin systems," in Proc. IEEE AP-S Int. Symp., sec. 61, July 7, 2001, pp. 109-112.

[21] Q. W. Pan and J. E. Allnutt, "Seasonal and diurnal effects on Ku-band site-diversity performance measured in a rainy tropical region," in Proc. IEEE AP-S Int. Symp., sec. 61, July 7, 2001, pp. 113-116.

[22] K.-T. Lin and C. A. Levis, "Site diversity for satellite earth terminals and measurements at $28 \mathrm{GHz}$," Proc. IEEE, vol. 81, pp. 897-904, June 1993.

[23] E. Matricciani and M. Mauri, "Italsat-Olympus 20-GHz orbital diversity experiment at spino," IEEE Trans. Antennas Propagat., vol. 43, pp. 105-108, Jan. 1995.

[24] T. Hatsuda, N. Okuno, H. Narita, K. Nishiura, and K. Kudo, "Systematic considerations of multiple satellites diversity on $14 / 12 \mathrm{GHz}$ band in snowing climates," in Proc. Int. Symp. Antennas and Propagation, Sept. 9, 1996, pp. 573-576.

[25] T. Hatsuda, N. Okuno, and R. Mituhashi, "Multiple satellites diversity characteristics in snowing climate and system application for 14/12 GHz band," in Proc. IEEE AP-S Int. Symp., sec. 81, June 6, 1998, pp. 2116-2119.

[26] T. Hatsuda, N. Okuno, K. Inoue, and K. Itoh, "Comparison of multiple satellites diversity characteristics between rain and snow attenuation for 14/12 GHz band," in Proc. IEEE AP-S Int. Symp., sec. 138, July 7, 1997, pp. 2556-2559.

[27] T. Hatsuda, N. Okuno, H. Nagase, K. Kojyou, K. Kojima, Y. Aoki, F. Takahata, and H. Echigo, "Long distance site diversity characteristics measurement results via JCSAT by using one earth station measuring method," in Proc. IEEE AP-S Int. Symp., sec. 5, July 1996, pp. $1872-1875$.

[28] T. Hatsuda and N. Okuno, "Long distance site diversity characteristics and effects by typhoon passing in $14 / 12 \mathrm{GHz}$ band satellite communication system," in Proc. 25th General Assembly Int. Union Radio Science (URSI-GA), vol. C8.2, Aug. 1996, pp. 157-157.
[29] T. Hatsuda et al., "Long distance site-diversity (SD) characteristics by using new measuring system," in Proc. IEEE AP-S Int. Symp., sec. 13, 07, 1998, pp. 408-411.

[30] _ _ "Theoretical and experimental consideration for $14 / 12 \mathrm{GHz}$ band signal attenuation by rain and snow clouds," in Proc. 26th General Assembly Int. Union Radio Science (URSI-GA), vol. FP.24, June 08, 1999, pp. 378-378.

[31] "Project COST 205: Frequency and Polarization Scalling of Rain Attenuation," Alta Frequenza, Special Issue on the COST 205 project on earth-satellite radio propagation above $10 \mathrm{GHz}$, vol. LIV, no. 3, pp. 157-172, May-June 1985

[32] T. Hatsuda, Y. Morihiro, S. Nakajima, Y. Nakamae, S. Mori, and T. Masamura, "The link calculation method of K-band satellite communication systems," Electrical Communication Lab. Tech. J., vol. 29, no. 4, pp. 135-152, 1980.

[33] "Specific Attenuation Model for Rain for Use in Prediction Methods,", Geneva, ITU-R Recommendation P.838-1, 1999.

[34] "Propagation Data and Prediction Methods Required for the Design of Earth-Space Telecommunication Systems,", Geneva, ITU-R Recommendation P.618-7, 2001.

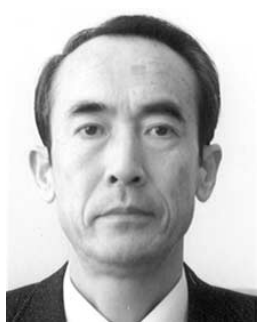

Takeshi Hatsuda (M'68) was born in Shizuoka, Japan, on December 2, 1941. He received the B.S., M.S., and Ph.D. degrees in electronics engineering, from the University of Hokkaido, Sapporo, Japan, in 1965, 1967, and 1974, respectively.

In 1967, he joined the Electrical Communication Laboratories, Nippon Telegraph and Telephone (NTT) Corporation, Yokosuka-shi, Japan, where he was Senior Research Engineer of NTT Radio Communication Networks Laboratories. Since 1987, he has been a Professor of the Department of Applied Electronics Engineering, Hokkaido Institute of Technology, where, since April 1998, he has been a Member of the Senate. His research activities are in the area of the transmission lines analysis, microwave integrated circuits, system design for the $30 / 20 \mathrm{GHz}$ band satellite communication systems, efficient utilization of the geostationary satellite orbit, and VSAT communication network and its applications.

Dr. Hatsuda is a Member of the Institute of Electronics, Information and Communication Engineers (IEICE) of Japan, the Institute of Image Information and Television Engineers (ITE) of Japan, the Institute of Professional Engineers of Japan (IPEJ), and the Digital Satellite Communication Universities Corporation Research Organization (Un-SAT). He received the 1990 Telecommunication System Technology Award by the Telecommunication Advancement Foundation.

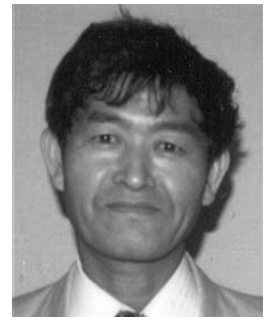

Yoshinao Aoki (M'66) received the B.E., M.E., and Ph.D. degrees in electronics engineering from Hokkaido University, Sapporo, Japan, in 1964, 1966, and 1972 respectively.

From 1967 to 1979, he was an Assistant Professor and since 1979, a professor of electronics and information engineering at Hokkaido University. From 1969 to 1971, he was a Visiting Scientist at Laval University, Quebec City, Ste-Foy, Canada. He is an author of Digital Signal Processing by Operator Method with Practical Processing Examples by $C$ Language and MATLAB (in Japanese) (Tokyo, Japan: Corona Publishing, 1996) and Lectures on Computer Graphics, (in Japanese) (Tokyo, Japan: Corona Publishing, 1997). His research interests include signal and image processing, computer graphics, and application of a communications satellite.

He is a Fellow of the Institute of Electronics, Information and Communication Engineers (IEICE) of Japan, a Member of the Information Processing Society of Japan (IPSJ), and the Digital Satellite Communication Universities Corporation Research Organization (Un-SAT). 


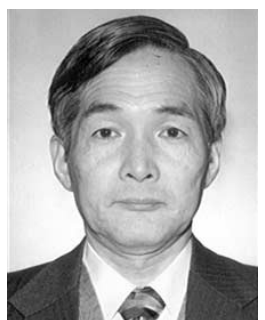

Hiroshi Echigo (S'68-M'71) received the B.E., M.E., and D.E. degrees in electrical communication engineering from Tohoku University, Tagajo, Japan, in 1966,1968 , and 1973 , respectively.

From 1973 to 1988, he was an Assistant Professor and then an Associate Professor in the Department of Electrical Communication Engineering, Tohoku University. While an Assistant Professor, he was involved in in one of the joint projects between Tohoku University and the University of Hawaii relating to the ALOHA Satellite Communication System for computer networks. Since 1988, he has been a Professor in the Department of Electrical Engineering and Information Technology, Tohoku Gakuin University, where works on EMC and satellite communications. From 1994 to 1998, he worked on Tohoku Gakuin University's Joint Study Group for Digital Satellite Communication.

Dr. Echigo is a Member of the Institute of Electronics, Information and Communication Engineers (IEICE) of Japan, the Institute of Electrical Engineers (IEE) of Japan, Image Information and Television Engineers (ITE) Japan, and the Digital Satellite Communication Universities Corporation Research Organization (Un-SAT).

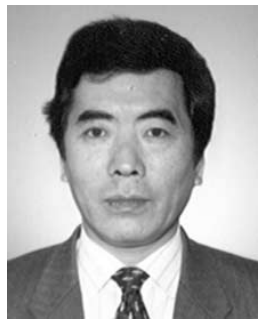

Fumio Takahata (M'80) received the B.Eng., M.Eng., and D.Eng. degrees from Waseda University, Tokyo, Japan, in 1972, 1974, and 1980, respectively.

He joined the KDD R\&D Laboratories, Tokyo, in 1974, where he was engaged in the research and development of digital satellite communications systems. Since 1988, he has been a Professor in the School of Science and Engineering, Waseda University. His research interests are in digital wireless communications.

Dr. Takahata is a Member of the Digital Satellite Communication Universities Corporation Research Organization (Un-SAT)

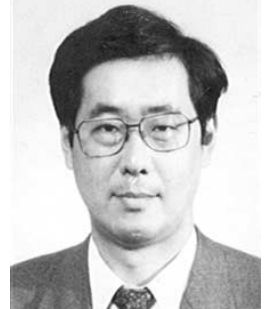

Yasuyuki Maekawa (M'87) received the B.S., M.S., and Ph.D. degrees in electronics from Kyoto University, Kyoto, Japan, in 1979, 1981, and 1985, respectively.

In 1985, he joined the Department of Telecommunication Engineering, Osaka Electro-Communication University, Neyagawa, Japan, where he is currently a Professor of satellite communication engineering. He has been engaged in Japan's CS-2 and CS-3 (domestic satellites) Pilot Programs, and satellite communication experiments using JCSAT among universities in Japan. His research activities are concerned with microwave and millimeter-wave propagation on the satellite-to-groun path and meteorological radar observation of hydrometeors, including dual polarization or multifrequency radar observation of thunderstorms.

Dr. Maekawa is a Member of the Institute of Electronics, Information and Communication Engineers (IEICE) of Japan, the Society of Geomagnetism and Earth Planetary and Space Sciences, the Meteorological Society of Japan, and the Digital Satellite Communication Universities Corporation Research Organization (Un-SAT).

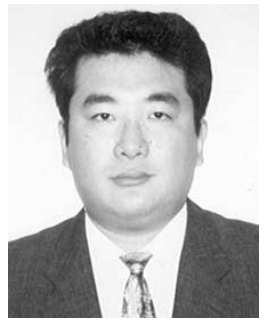

Kiyotaka Fujisaki received the B.E. degree in electrical engineering from Kagoshima University, Japan, in 1988 and the M.E. and D.E. degrees in computer science and communication engineering from Kyushu University, Fukuoka, Japan, in 1990 and 1996, respectively.

He joined the Faculty of Engineering, Kyushu University, as a Research Associate in 1993, where he is currently an Associate Professor. His research interests are satellite communications and remote sensing.

Dr. Fujisaki is a Member of the Institute of Electronics, Information and Communication Engineers (IEICE) of Japan and the Digital Satellite Communication Universities Corporation Research Organization (Un-SAT). 